\title{
A COMPACT SYMMETRIC SYMPLECTIC NON-KAEHLER MANIFOLD: REVISIT
}

\author{
MiN KYU KIM
}

\begin{abstract}
Lerman constructed a twelve dimensional Hamiltonian circle action with an isolated fixed point on a non-Kaehler manifold. In this report, the author constructs such an example which is eight dimensional.
\end{abstract}

\section{Introduction}

In [Ler], Lerman constructed a twelve dimensional Hamiltonian circle action with an isolated fixed point on a non-Kaehler manifold. In this report, the author constructs an eight dimensional semifree Hamiltonian circle action with an isolated fixed point on a simply connected non-Kaehler manifold. The example raises the following question: Can we construct such an example which is six dimensional?

\section{Construction}

Gompf constructed a six dimensional simply connected symplectic nonKaehler manifold $M^{6}$ such that $M^{6} \times S^{2}$ is also non-Kaehler [Gom, Theorem 7.1]. He shows that there exists a nontrivial element $q$ in $H^{2}\left(M^{6} \times S^{2}\right)$ such that $q \wedge w^{2}=0$ for all $w$ in $H^{2}\left(M^{6} \times S^{2}\right)$. Hence by the Hard Lefschetz Theorem, the manifold $M^{6} \times S^{2}$ (also $M^{6} \times S^{2} \# \overline{\mathbb{C} P^{4}}$ ) is non-Kaehler.

We give the trivial circle action on $M^{6}$ and the usual rotation on $S^{2}$. Hence the manifold $M^{6} \times S^{2}$ has a Hamiltonian circle action with two copies of $M^{6}$ as the fixed set. If we blow up $M^{6} \times S^{2}$ at a fixed point with the action of weight $(1,0,0,0)$, then a simple computation shows that the blown up manifold has an isolated fixed point with the action of weight $(1,-1,-1,-1)$. Also, it is non-Kaehler since it is diffeomorphic to $M^{6} \times S^{2} \# \overline{\mathbb{C} P^{4}}$.

\section{References}

[Gom] R. E. Gompf, A new construction of symplectic manifolds, Ann. of Math. 142 (1995), 527-595.

[Ler] E. Lerman, A compact symmetric symplectic non-Kaehler manifold, Math. Res. Lett. 3 (1996), 587-590.

Received February 26, 2002.

This work was supported by BK21 Project, KAIST, in 2002.

Key words and phrases. Hamiltonian action, non-Kaehler manifold. 
Department of Mathematics, Korea Advanced Institute of Science and TechnolOGY, KOREA.

E-mail address: minkyu@math.kaist.ac.kr 